\title{
Finite Fractal Dimensionality of Compact Kernel Sections for Dissipative Non-Autonomous Klein-Gordon-Schrödinger Lattice Systems
}

\author{
Jinwu Huang \\ Department of Mathematics, Shanghai Normal University Tianhua College, Shanghai, China \\ Email: eyesonme_hmily@msn.com
}

How to cite this paper: Huang, J.W. (2020) Finite Fractal Dimensionality of Compact Kernel Sections for Dissipative Non-Autonomous Klein-Gordon-Schrödinger Lattice Systems. Journal of Applied Mathematics and Physics, 8, 2919-2929. https://doi.org/10.4236/jamp.2020.812215

Received: October 3, 2020

Accepted: December 15, 2020

Published: December 18, 2020

Copyright $\odot 2020$ by author(s) and Scientific Research Publishing Inc. This work is licensed under the Creative Commons Attribution International License (CC BY 4.0).

http://creativecommons.org/licenses/by/4.0/ In recent years, great progress had been made in the study of non-autonomous (c) (i) Open Access

\begin{abstract}
In this paper, an upper bound of fractal dimension of the compact kernel sections for the dissipative non-autonomous Klein-Gordon-Schrödinger lattice system is obtained, by applying a criterion for estimating fractal dimension of a family of compact subsets of a separable Hilbert space.
\end{abstract}

\section{Keywords}

Compact Kernel Sections, Dissipative, Fractal Dimension, Non-Autonomous, Klein-Gordon-Schrödinger Lattice System

\section{Introduction} infinite dimensional dynamical systems. See, e.g., [1] [2] [3] [4] [5] and the references therein. Lattice dynamical systems (Hereafter LDSs) are infinite dimensional ordinary differential equations, which were widely and deeply investigated in the past decades due to its wide application in many fields such as laser systems, material science, electrical engineering, biology, chemical reaction theory and etc. See, e.g., [6]-[17] and so on. Nowadays, the study of non-autonomous LDSs appealed to more and more researchers, but there are few papers for non-autonomous LDSs until now. See e.g., [18]-[23] and etc.

As to the dissipative autonomous Klein-Gordon-Schrödinger (Hereafter KGS) lattice systems, many authors have studied them. For example, Abdallah in [24], Abounouh, Goubet and Hakim in [25], Yin and Zhou et al. in [26] investigated the existence, regularity, upper semicontinuity, Kolmogorov entropy of global 
attractor and so forth. Meanwhile, the following dissipative non-autonomous KGS lattice system

$$
\left\{\begin{array}{l}
i \dot{w}_{m}-\left(w_{m-1}+w_{m+1}-2 w_{m}\right)+i \alpha w_{m}+u_{m} w_{m}=f_{m}(t), \\
\ddot{u}_{m}+\beta \dot{u}_{m}+\left(u_{m-1}+u_{m+1}-2 u_{m}\right)+\gamma u_{m}-\mu\left|w_{m}\right|^{2}=g_{m}(t),
\end{array} \quad m \in \mathbf{Z}, t>\tau \in \mathbf{R}\right.
$$

was investigated by many researchers either. Specifically, the existence of uniform exponential attractors for the dissipative non-autonomous KGS lattice system (1.1) with quasi-periodic symbols is studied in weighted spaces of infinite sequences by Abdallah in [27], simultaneously, some main results that the solution semigroup associated with such a system is Lipschitz continuous, $\alpha$-contraction and satisfies the squeezing property, are obtained under some premise. Huang et al. in [28] proved the existence of a compact uniform attractor and obtained an upper bound of the Kolmogorov entropy of the compact uniform attractor. In addition, an upper semicontinuity of the compact uniform attractor is established as well. Zhao and Zhou in [29] proved the existence of compact kernel sections and obtained an upper bound of the Kolmogorov entropy of the compact kernel sections, but they didn't study the fractal dimension of the compact kernel sections. In Zhou and Han [30], some sufficient conditions for the existence of a uniform exponential attractor for a family of continuous processes on separable Hilbert spaces and the space of infinite sequences are presented at first, and then the existence of uniform exponential attractors for the dissipative non-autonomous KGS lattice system (1.1) and for the dissipative non-autonomous Zakharov lattice system driven by quasi-periodic external forces in the spaces of infinite sequences is studied. However, what's more important, so far to our knowledge, this problem that the fractal dimension of the compact kernel sections was not studied in Zhao and Zhou [29] is still an open topic till today. In view of this point, this paper is to estimate the fractal dimension of the compact kernel sections for the dissipative non-autonomous KGS lattice system (1.1). For our purpose, we first mention that as we all know, if $\mathcal{A}$ is a compact set in a metric space such that the fractal dimension of $\mathcal{A}$ is less or equal to $n / 2$ for some $n \in \mathbf{N}$, then there exists an injective Lipschitz mapping $\mathscr{F}: \mathcal{A} \rightarrow \mathbf{R}^{n}$ such that its inverse is Hölder continuous. In the sequel of this paper, we will present a criterion for estimating the fractal dimension of a family of compact subsets of a separable Hilbert space and then apply this criterion to obtain an upper bound of the fractal dimension of the compact kernel sections associated with the dissipative non-autonomous KGS lattice system (1.1).

The remaining of this paper is organized as below. We give the preliminaries in Section 2. In Sections 3, a criterion is used to estimate the fractal dimension of the compact kernel sections for the dissipative non-autonomous KGS lattice system, and an upper bound is obtained. Lastly, Section 4 presents the conclusions.

\section{Preliminaries}

To begin, we introduce 


$$
\begin{aligned}
& \ell^{2}=\left\{x=\left(x_{m}\right)_{m \in \mathbf{Z}} \mid x_{m} \in \mathbf{R}, \sum_{m \in \mathbf{Z}} x_{m}^{2}<+\infty\right\}, \\
& l^{2}=\left\{x=\left.\left(x_{m}\right)_{m \in \mathbf{Z}}\left|x_{m} \in \mathbf{C}, \sum_{m \in \mathbf{Z}}\right| x_{m}\right|^{2}<+\infty\right\},
\end{aligned}
$$

where $\mathbf{Z}, \mathbf{R}$ and $\mathbf{C}$ denote the integral, real and complex numbers, respectively.

Write $H=\ell^{2}$ or $l^{2}$, and endow $H$ with the inner product and norm as below

$$
(x, y)=\sum_{m \in \mathbf{Z}} x_{m} \bar{y}_{m}, \quad\|x\|^{2}=(x, x), \quad \forall x=\left(x_{m}\right)_{m \in \mathbf{Z}}, \quad y=\left(y_{m}\right)_{m \in \mathbf{Z}} \in H,
$$

where $\bar{y}_{m}$ is the conjugate of $y_{m}$. Clearly, $H$ is a Hilbert space.

Define linear operators $A$ and $B$ as follows

$$
(A x)_{m}=2 x_{m}-x_{m-1}-x_{m+1}, \quad(B x)_{m}=x_{m+1}-x_{m}, \quad \forall x=\left(x_{m}\right)_{m \in \mathbf{Z}} \in H .
$$

For any $x, y \in H$, define a bilinear form by means of

$$
(x, y)_{\gamma}=(B x, B y)+\gamma(x, y),
$$

where $\gamma$ as in the dissipative non-autonomous KGS lattice system (1.1) presented above. This bilinear form is obviously an inner product in Hilbert space $H$.

In the end, we express Hilbert spaces $\ell_{\gamma}^{2}, \ell^{2}$ and $l^{2}$ as

$$
\ell_{\gamma}^{2}=\left(\ell^{2},(\cdot, \cdot)_{\gamma},\|\cdot\|_{\gamma}\right), \quad \ell^{2}=\left(\ell^{2},(\cdot, \cdot),\|\cdot\|\right), \quad l^{2}=\left(l^{2},(\cdot, \cdot),\|\cdot\|\right) .
$$

Set

$$
H_{\gamma}=\ell_{\gamma}^{2} \times \ell^{2} \times l^{2},
$$

and equip it with the following norm and inner product

$$
\begin{gathered}
\|\varphi\|_{H_{\gamma}}^{2}=(\varphi, \varphi)_{H_{\gamma}}, \quad \forall \varphi \in H_{\gamma} ; \\
\left(\varphi^{(1)}, \varphi^{(2)}\right)_{H_{\gamma}}=\sum_{m \in \mathbf{Z}}\left(\left(B u^{(1)}\right)_{m}\left(B u^{(2)}\right)_{m}+\gamma u_{m}^{(1)} u_{m}^{(2)}+v_{m}^{(1)} v_{m}^{(2)}+w_{m}^{(1)} \overline{w_{m}^{(2)}}\right),
\end{gathered}
$$

where $\varphi^{(l)}=\left(u^{(t)}, v^{(t)}, w^{(l)}\right)_{m \in \mathbf{Z}} \in H_{\gamma}, l=1,2$.

Define

$$
\begin{aligned}
\mathcal{M}=\left\{h(t)=\left(h_{m}(t)\right)_{m \in \mathbf{Z}} \in \mathcal{C}_{b}(\mathbf{R}, H): \text { for each } \tau \in \mathbf{R} \text { and } \forall \epsilon>0,\right. \\
\left.\exists \mathcal{N}(\epsilon) \in \mathbf{N} \text { such that } \sum_{|m| \geq \mathcal{N}(\epsilon)}\left|h_{m}(\imath)\right|^{2} \leq \epsilon \text { for any } \imath \leq \tau\right\},
\end{aligned}
$$

and denote by $\mathcal{C}_{b}\left(\mathbf{R}, \ell^{2}\right)$ and $\mathcal{C}_{b}\left(\mathbf{R}, l^{2}\right)$ respectively the set of continuous and bounded functions from $\mathbf{R}$ into $\ell^{2}$ and $l^{2}$.

Definition 2.1. A two-parameter family of mappings $\{U(t, \tau)\}_{t \geq \tau}$ is called to be a process in a Hilbert space $\mathscr{C}$, if
1) $U(t, \tau): \mathscr{H} \rightarrow \mathscr{H}, t \geq \tau$;
2) $U(t, s) U(s, \tau)=U(t, \tau), \forall t \geq s \geq \tau, \tau \in \mathbf{R}$; 
3) $U(\tau, \tau)=I$ (identity operator of $\mathscr{H}$ ), $\tau \in \mathbf{R}$.

Definition 2.2. A function $\varphi(s), s \in \mathbf{R}$, is said to be a complete trajectory of the process $\{U(t, \tau)\}_{t \geq \tau}$, if $U(t, \tau) \varphi(\tau)=\varphi(t), \forall t \geq \tau, \tau \in \mathbf{R}$. The kernel $\mathcal{K}$ of the process $\{U(t, \tau)\}_{t \geq \tau}$ consists of all bounded complete trajectories of $\{U(t, \tau)\}_{t \geq \tau}$, i.e.,

$$
\mathcal{K}=\left\{\varphi(\cdot): U(t, \tau) \varphi(\tau)=\varphi(t), t \geq \tau, \tau \in \mathbf{R},\|\varphi(s)\|_{\mathscr{H}} \leq \mathcal{M}_{\varphi}, s \in \mathbf{R}\right\},
$$

and the kernel sections $\mathcal{K}(s) \subset \mathscr{K}$ of the kernel $\mathcal{K}$ at time $s \in \mathbf{R}$ is

$$
\mathcal{K}(s)=\{\varphi(s): \varphi(\cdot) \in \mathcal{K}\} .
$$

Definition 2.3. The fractal dimension $\operatorname{dim}_{f} \mathcal{A}$ of a compact set $\mathcal{A}$ in a metric space $\mathcal{H}$ is defined as follows, namely

$$
\operatorname{dim}_{f} \mathcal{A}=\lim _{\varepsilon \rightarrow 0} \sup \frac{\ln N(\mathcal{A}, \varepsilon)}{\ln (1 / \varepsilon)},
$$

where $N(\mathcal{A}, \varepsilon)$ is the minimal number of closed sets of radius $\varepsilon$ which cover the set $\mathcal{A}$.

The criterion below is directly cited from Zhou et al. [21].

Lemma 2.1. Let $\{U(t, \tau)\}_{t \geq \tau}$ be a continuous process on a Hilbert space and $\{\mathscr{d}(t)\}_{t \in \mathbf{R}}$ be a family of compact, negatively invariant (i.e.,

$\mathscr{d}(t) \subset U(t, \tau) . /(\tau)$ for all $t \geq \tau, \tau \in \mathbf{R})$ subsets of $\mathscr{H}$. Assume that

1) there exists a uniform finite covering of closed subsets with diameter 2 of

$\mathscr{d}(t)$ for all $t \in \mathbf{R}$, that is, there exists $N^{\star}$ closed balls of $\mathcal{M}$ with diameter 2 covering $\mathscr{C}(t)$ for all $t \in \mathbf{R}$, where $N^{\star}$ is independent of $t$;

2) for any $\tau \in \mathbf{R}$, there exists $\Gamma>0$ and $0<\eta<1$, which are all independent of $\tau \in \mathbf{R}$ such that for $\omega_{\imath} \in \mathscr{C}(\tau), \imath=1,2$,

a) there exists $L>0$ yields

$$
\left\|U(\Gamma+\tau, \tau) \omega_{1}-U(\Gamma+\tau, \tau) \omega_{2}\right\|_{\mathscr{R}} \leq L\left\|\omega_{1}-\omega_{2}\right\|_{\not{H}},
$$

i.e., $U(\Gamma+\tau, \tau)$ is Lipschitz on $\mathscr{C}(\tau)$;

b) there exists finite-dimensional orthoprojector $P$ of $\mathscr{H}$ satisfies

$$
\left\|(I-P)\left(U(\Gamma+\tau, \tau) \omega_{1}-U(\Gamma+\tau, \tau) \omega_{2}\right)\right\|_{\mathscr{H}} \leq \eta\left\|\omega_{1}-\omega_{2}\right\|_{\mathscr{M}} ;
$$

then

$$
\operatorname{dim}_{f} \mathscr{C} \leq \operatorname{dim} P \cdot \ln \left(1+\frac{8(1+L)}{1-\eta}\right) \cdot\left(\ln \frac{2}{1+\eta}\right)^{-1}
$$

\section{Fractal Dimension of Compact Kernel Sections for Dissipative Non-Autonomous KGS Lattice System}

Consider the dissipative non-autonomous KGS lattice system with the initial conditions as vector form

$$
\left\{\begin{array}{l}
i \dot{w}-A w+i \alpha w+u w=f(t), \\
\ddot{u}+\beta \dot{u}+A u+\gamma u-\mu|w|^{2}=g(t), \quad t>\tau, \quad \tau \in \mathbf{R} \\
w(\tau)=\left(w_{m}(\tau)\right)_{m \in \mathbf{Z}}, \quad u(\tau)=\left(u_{m}(\tau)\right)_{m \in \mathbf{Z}}, \quad \dot{u}(\tau)=\left(\dot{u}_{m}(\tau)\right)_{m \in \mathbf{Z}},
\end{array}\right.
$$


where $\quad w=\left(w_{m}\right)_{m \in \mathbf{Z}}, \quad w_{m} \in \mathbf{C} ; \quad u=\left(u_{m}\right)_{m \in \mathbf{Z}}, \quad u_{m} \in \mathbf{R} ; \quad u w=\left(u_{m} w_{m}\right)_{m \in \mathbf{Z}}$, $|w|^{2}=\left(\left|w_{m}\right|^{2}\right)_{m \in \mathbf{Z}} ; f(t)=\left(f_{m}(t)\right)_{m \in \mathbf{Z}}, g(t)=\left(g_{m}(t)\right)_{m \in \mathbf{Z}} ; i$ is the imaginary numbers' unit; $\alpha, \beta, \gamma$ and $\mu$ are positive constants;

$(A z)_{m}=2 z_{m}-z_{m-1}-z_{m+1}, m \in \mathbf{Z}, z$ denotes $u$ or $w$.

We set

$$
v=\dot{u}+\lambda u, \quad \lambda=\frac{\beta \gamma}{\beta^{2}+4 \gamma}>0 .
$$

Thus, (3.1) can be written as below

$$
\left\{\begin{array}{l}
\dot{\varphi}+G \varphi=F(\varphi, t), \quad t>\tau ; \\
\varphi(\tau)=(u(\tau), v(\tau), w(\tau))^{\mathrm{T}}=(u(\tau), \dot{u}(\tau)+\lambda u(\tau), w(\tau))^{\mathrm{T}}, \quad \tau \in \mathbf{R} ;
\end{array}\right.
$$

where $\varphi=(u, v, w)^{\mathrm{T}}, \quad v=\dot{u}+\lambda u, \quad F(\varphi, t)=\left(0, \mu|w|^{2}+g(t), i u w-i f(t)\right)^{\mathrm{T}}$, and

$$
G=\left(\begin{array}{ccc}
\lambda I & -I & 0 \\
A+\gamma I+\lambda(\lambda-\beta) I & \beta-\lambda & 0 \\
0 & 0 & i A+\lambda I
\end{array}\right)
$$

From Zhao and Zhou [29], we can see, for given $f(t)=\left(f_{m}(t)\right)_{m \in \mathbf{Z}} \in \mathcal{M}$ with $H=l^{2}, g(t)=\left(g_{m}(t)\right)_{m \in \mathbf{Z}} \in \mathcal{M}$ with $H=\ell^{2}$, the solution mappings of (3.2), that is,

$U(t, \tau): \varphi(\tau)=(u(\tau), v(\tau), w(\tau))^{\mathrm{T}} \in H_{\gamma} \rightarrow \varphi(t)=(u(t), v(t), w(t))^{\mathrm{T}} \in H_{\gamma}$, $\forall t \geq \tau, \tau \in \mathbf{R}$, generate a family of continuous processes $\{U(t, \tau)\}_{t \geq \tau}$ in $H_{\gamma}$. Moreover, the family of processes $\{U(t, \tau)\}_{t \geq \tau}, \tau \in \mathbf{R}$, possess a family of compact kernel sections $\{\mathcal{K}(\tau)\}_{\tau \in \mathbf{R}}$, where $\mathcal{K}(\tau)$ is included in a uniformly bounded set $\mathscr{B}=\left\{\varphi \in H_{\gamma}:\|\varphi\|_{H_{\gamma}}^{2} \leq R_{0}^{2}\right\}$ and satisfies

$$
\begin{aligned}
U(t, \tau) \mathcal{K}(\tau) & =\mathcal{K}(t), \forall t \geq \tau, \tau \in \mathbf{R} \text {, here } \\
R_{0} & =\left(\frac{1}{\delta_{0}}\left(\frac{\sup _{t \in \mathbf{R}}\|f(t)\|^{2}}{\alpha}+\frac{\sup _{t \in \mathbf{R}}\|g(t)\|^{2}}{\beta}+\frac{2 \mu^{2} \sup _{t \in \mathbf{R}}\|f(t)\|^{4}}{\alpha^{4} \beta}\right)\right)^{1 / 2}, \\
\delta_{0} & =\min \left\{\delta, \frac{\alpha}{4}\right\}, \quad \delta=\frac{\beta \gamma}{\sqrt{\beta^{2}+4 \gamma}\left(\sqrt{\beta^{2}+4 \gamma}+\beta\right)}>0 .
\end{aligned}
$$

In the sequel, we get an upper bound of the fractal dimension of the compact kernel sections $\mathcal{K}(\tau)$, which is generated by the process of the dissipative non-autonomous KGS lattice system (3.1).

Suppose $\varphi^{(t)}(\tau) \in \mathcal{K}(\tau), \quad \tau \in \mathbf{R}$, then $\varphi^{(t)}(t)=U(t, \tau) \varphi^{(t)}(\tau)=\left(u^{(t)}(t), v^{(t)}(t), w^{(t)}(t)\right)^{\mathrm{T}} \in \mathcal{K}(\tau) \subseteq \mathscr{B}$ for $t-\tau \geq T(\tau, \mathscr{B}), \quad \imath=1,2$. Set $\phi(t)=\varphi^{(1)}(t)-\varphi^{(2)}(t)$, then by (3.2), we have

$$
\left\{\begin{array}{l}
\dot{\phi}+G \phi=F\left(\varphi^{(1)}, t\right)-F\left(\varphi^{(2)}, t\right), \\
\phi(\tau)=(\xi(\tau), \zeta(\tau), \varsigma(\tau))^{\mathrm{T}},
\end{array} \quad t>\tau, \quad \tau \in \mathbf{R},\right.
$$

where $\phi=(\xi, \zeta, \varsigma)^{\mathrm{T}}=\left(\xi_{m}, \zeta_{m}, \varsigma_{m}\right)_{m \in \mathbf{Z}}^{\mathrm{T}}=\left(\phi_{m}\right)_{m \in \mathbf{Z}}$, and $\xi=u^{(1)}-u^{(2)}$, 
$\zeta=\dot{\xi}+\lambda \xi=v^{(1)}-v^{(2)}, \quad \varsigma=w^{(1)}-w^{(2)}$.

Lemma 3.1. For any $\Gamma>0, U(\Gamma+\tau, \tau)$ is Lipschitz on $\mathcal{K}(\tau)$, i.e.,

$$
\left\|\varphi^{(1)}(\Gamma+\tau)-\varphi^{(2)}(\Gamma+\tau)\right\|_{H_{\gamma}} \leq \mathrm{e}^{\left(C_{0} R_{0}^{2}-\delta_{0}\right) \Gamma}\left\|\varphi^{(1)}(\tau)-\varphi^{(2)}(\tau)\right\|_{H_{\gamma}},
$$

where

$$
C_{0}=\frac{2 \mu^{2}}{\beta}+\frac{1}{3 \alpha}
$$

$R_{0}$ and $\delta_{0}$ as in (3.3) and (3.4), respectively.

For brief, we denote by $\operatorname{Re}(\cdot, \cdot)$ and $\operatorname{Im}(\cdot, \cdot)$ respectively the real part and imaginary part of inner product $(\cdot, \cdot)$.

Proof. Taking the real part of the inner product $(\cdot, \cdot)_{H_{\gamma}}$ of (3.5) with $\phi$, we have

$$
\operatorname{Re}(\dot{\phi}, \phi)_{H_{\gamma}}+\operatorname{Re}(G \phi, \phi)_{H_{\gamma}}=\operatorname{Re}\left(F\left(\varphi^{(1)}, t\right)-F\left(\varphi^{(2)}, t\right), \phi\right)_{H_{\gamma}} .
$$

By simple computation, we get

$$
\begin{gathered}
\operatorname{Re}(\dot{\phi}, \phi)_{H_{\gamma}}=\frac{1}{2} \frac{\mathrm{d}}{\mathrm{d} t}\|\phi(t)\|_{H_{\gamma}}^{2}, \\
\operatorname{Re}(G \phi, \phi)_{H_{\gamma}} \geq \delta\left(\|\xi\|_{\gamma}^{2}+\|\zeta\|^{2}\right)+\frac{\beta}{2}\|\zeta\|^{2}+\alpha\|\varsigma\|^{2} \\
\geq \delta_{0}\|\phi\|_{H_{\gamma}}^{2}+\frac{\beta}{2}\|\zeta\|^{2}+\frac{3 \alpha}{4}\|\varsigma\|^{2}, \\
\operatorname{Re}\left(F\left(\varphi^{(1)}, t\right)-F\left(\varphi^{(2)}, t\right), \phi\right)_{H_{\gamma}} \\
\leq \frac{\beta}{2}\|\zeta\|^{2}+\frac{3 \alpha}{4}\|\varsigma\|^{2}+\left(\frac{\mu^{2}}{2 \beta}+\frac{1}{12 \alpha}\right)\|\phi\|_{H_{\gamma}}^{2}\left(\left|w^{(1)}\right|+\left|w^{(2)}\right|^{2} .\right.
\end{gathered}
$$

Actually

$$
\begin{aligned}
& \operatorname{Re}\left(F\left(\varphi^{(1)}, t\right)-F\left(\varphi^{(2)}, t\right), \phi\right)_{H_{\gamma}} \\
& =\operatorname{Re} \mu\left(\left|w^{(1)}\right|^{2}-\left|w^{(2)}\right|^{2}, \zeta\right)+\operatorname{Re}\left(i\left(u^{(1)} w^{(1)}-u^{(2)} w^{(2)}\right), \varsigma\right) \\
& \leq \mu\|\zeta\|\|\varsigma\|\left(\left|w^{(1)}\right|+\left|w^{(2)}\right|\right)+\operatorname{Re}\left(i\left(u^{(1)} w^{(1)}-u^{(2)} w^{(2)}\right), \varsigma\right),
\end{aligned}
$$

and

$$
\begin{aligned}
& \operatorname{Re}\left(i\left(u^{(1)} w^{(1)}-u^{(2)} w^{(2)}\right), \varsigma\right) \\
& =\operatorname{Re}\left(i \frac{\left(u^{(1)}-u^{(2)}\right)\left(w^{(1)}+w^{(2)}\right)+\left(u^{(1)}+u^{(2)}\right)\left(w^{(1)}-w^{(2)}\right)}{2}, \varsigma\right) \\
& =-\operatorname{Im}\left(\frac{\left(u^{(1)}-u^{(2)}\right)\left(w^{(1)}+w^{(2)}\right)}{2}, \varsigma\right) \\
& \leq \frac{1}{2}\|\xi\|_{\gamma}\|\varsigma\|\left(\left|w^{(1)}\right|+\left|w^{(2)}\right|\right),
\end{aligned}
$$


thus

$$
\begin{aligned}
& \operatorname{Re}\left(F\left(\varphi^{(1)}, t\right)-F\left(\varphi^{(2)}, t\right), \phi\right)_{H_{\gamma}} \\
& \leq \mu\|\zeta\|\|\varsigma\|\left(\left|w^{(1)}\right|+\left|w^{(2)}\right|\right)+\frac{1}{2}\|\xi\|_{\gamma}\|\varsigma\|\left(\left|w^{(1)}\right|+\left|w^{(2)}\right|\right) .
\end{aligned}
$$

Applying Young's inequality to (3.12), it is obvious to know that (3.11) holds. Taking (3.8)-(3.11) into account, we see

$$
\begin{aligned}
\frac{\mathrm{d}}{\mathrm{d} t}\|\phi(t)\|_{H_{\gamma}}^{2} & \leq-2 \delta_{0}\|\phi(t)\|_{H_{\gamma}}^{2}+\left(\frac{\mu^{2}}{\beta}+\frac{1}{6 \alpha}\right)\|\phi(t)\|_{H_{\gamma}}^{2}\left(\left|w^{(1)}\right|+\left|w^{(2)}\right|\right)^{2} \\
& \leq 2\left(C_{0} R_{0}^{2}-\delta_{0}\right)\|\phi(t)\|_{H_{\gamma}}^{2} .
\end{aligned}
$$

Set $t=\Gamma+\tau, \Gamma>0$, and then apply Gronwall's inequality to (3.13), it is easy to see that (3.6) holds. The proof is completed.

Lemma 3.2. There exists a finite dimensional orthoprojector $\mathscr{P}$ of $H_{\gamma}$ and $\tilde{\eta} \in(0,1)$ such that

$$
\begin{aligned}
& \left\|(I-\mathscr{P})\left(U\left(\Gamma_{0}+\tau, \tau\right) \varphi^{(1)}(\tau)-U\left(\Gamma_{0}+\tau, \tau\right) \varphi^{(2)}(\tau)\right)\right\|_{H_{\gamma}} \\
& \leq \tilde{\eta}\left\|\varphi^{(1)}(\tau)-\varphi^{(2)}(\tau)\right\|_{H_{\gamma}} .
\end{aligned}
$$

Proof. For this purpose, we choose an increasingly smooth function $\chi(\theta) \in \mathcal{C}^{1}\left(\mathbf{R}^{+},[0,1]\right)$, yielding

$$
\left\{\begin{array}{lc}
\chi(\theta)=0, & 0 \leq \theta \leq 1 \\
0 \leq \chi(\theta) \leq 1, & 1 \leq \theta \leq 2 \\
\chi(\theta)=1, & \theta \geq 2
\end{array}\right.
$$

and at the same time, there exists a constant $\chi_{0}$ such that $\left|\chi^{\prime}(\theta)\right| \leq \chi_{0}$, $\forall \theta \in \mathbf{R}^{+}$.

Let $M$ be a fixed positive integer, set $\psi=\left(\psi_{m}\right)_{m \in \mathbf{Z}}=\left(\chi\left(\frac{|m|}{M}\right) \phi_{m}\right)_{m \in \mathbf{Z}}$, $\phi_{m}=\left(\xi_{m}, \zeta_{m}, \varsigma_{m}\right)_{m \in \mathbf{Z}}^{\mathrm{T}}$. Taking the real part of the inner product $(\cdot, \cdot)_{H_{\gamma}}$ in (3.5) with $\psi$, we get

$$
\operatorname{Re}(\dot{\phi}, \psi)_{H_{\gamma}}+\operatorname{Re}(G \phi, \psi)_{H_{\gamma}}=\operatorname{Re}\left(F\left(\varphi^{(1)}, t\right)-F\left(\varphi^{(2)}, t\right), \psi\right)_{H_{\gamma}} .
$$

Similar to Zhou [14], we have

$$
\boldsymbol{\operatorname { R e }}(\dot{\phi}, \psi)_{H_{\gamma}} \geq \frac{1}{2} \frac{\mathrm{d}}{\mathrm{d} t} \sum_{m \in \mathbf{Z}} \chi\left(\frac{|m|}{M}\right)\left\|\phi_{m}\right\|_{H_{\gamma}}^{2}+\frac{2 \chi_{0}(\gamma+\lambda+1)}{M \gamma}\|\phi\|_{H_{\gamma}}^{2},
$$

$$
\begin{aligned}
& \operatorname{Re}(G \phi, \psi)_{H_{\gamma}} \\
& \geq \sum_{m \in \mathbf{Z}} \chi\left(\frac{|m|}{M}\right)\left(\delta\left(\left\|\xi_{m}\right\|_{\gamma}^{2}+\left|\zeta_{m}\right|^{2}\right)+\frac{\beta}{2} \zeta_{m}^{2}+\alpha\left|\varsigma_{m}\right|^{2}\right)-\frac{\chi_{0}(3 \gamma+2 \lambda+2)}{M \gamma}\|\phi\|_{H_{\gamma}}^{2} \\
& \geq \sum_{m \in \mathbf{Z}} \chi\left(\frac{|m|}{M}\right)\left(\delta_{0}\left\|\phi_{m}\right\|_{H_{\gamma}}^{2}+\frac{\beta}{2} \zeta_{m}^{2}+\frac{3 \alpha}{4}\left|\zeta_{m}\right|^{2}\right)-\frac{\chi_{0}(3 \gamma+2 \lambda+2)}{M \gamma}\|\phi\|_{H_{\gamma}}^{2},
\end{aligned}
$$


and analogous to (3.11), we obtain

$$
\begin{aligned}
& \operatorname{Re}\left(F\left(\varphi^{(1)}, t\right)-F\left(\varphi^{(2)}, t\right), \psi\right)_{H_{\gamma}} \\
& \leq \sum_{m \in \mathbf{Z}} \chi\left(\frac{|m|}{M}\right)\left(\frac{\beta}{2} \zeta_{m}^{2}+\frac{3 \alpha}{4}\left|\varsigma_{m}\right|^{2}+\frac{C_{0}}{4}\left\|\phi_{m}\right\|_{H_{\gamma}}^{2}\left(\left|w_{m}^{(1)}\right|+\left|w_{m}^{(2)}\right|\right)^{2}\right) .
\end{aligned}
$$

Combining (3.15)-(3.18), we get

$$
\begin{aligned}
& \frac{\mathrm{d}}{\mathrm{d} t} \sum_{m \in \mathbf{Z}} \chi\left(\frac{|m|}{M}\right)\left\|\phi_{m}\right\|_{H_{\gamma}}^{2} \\
& \leq \sum_{m \in \mathbf{Z}} \chi\left(\frac{|m|}{M}\right)\left(-2 \delta_{0}\left\|\phi_{m}\right\|_{H_{\gamma}}^{2}+\frac{C_{0}}{2}\left\|\phi_{m}\right\|_{H_{\gamma}}^{2}\left(\left|w_{m}^{(1)}\right|+\left|w_{m}^{(2)}\right|\right)^{2}\right)+\frac{2 \chi_{0}}{M}\|\phi\|_{H_{\gamma}}^{2} .
\end{aligned}
$$

From Zhao and Zhou [29], we know that for $\eta_{0}=\frac{\delta_{0}}{2 C_{0}}>0$, there exist $\Gamma_{0}=\Gamma_{0}\left(\eta_{0}, \tau, \mathscr{\beta}\right) \geq T(\tau, \mathscr{\beta})$ and an integer $M\left(\eta_{0}, \tau, \mathscr{\beta}\right) \in \mathbf{N}$, which satisfies

$$
\frac{1}{2 \alpha} \sum_{|m| \geq M\left(\eta_{0}, \tau, \not \beta\right)}\left|f_{m}(\tau)\right|^{2}+\frac{1}{\beta} \sum_{|m| \geq M\left(\eta_{0}, \tau, \not ̈\right)} g_{m}(\tau)^{2}+\frac{\widetilde{C_{0}}}{M\left(\eta_{0}, \tau, \mathscr{\beta}\right)} \leq \frac{3 \delta_{0} \eta_{0}}{10}
$$

where

$$
\widetilde{C_{0}}=\frac{2 \mu^{2} \chi_{0} R_{0}^{4}}{\alpha \beta}+\frac{\chi_{0} R_{0}^{2}(5 \gamma+4 \lambda+4)}{\gamma}
$$

such that

$$
\sum_{|m| \geq 2 M}\left\|\varphi^{(t)}(t)\right\|_{H_{\gamma}}^{2} \leq \eta_{0}=\frac{\delta_{0}}{2 C_{0}}, \quad \imath=1,2, \forall M \geq M\left(\eta_{0}, \tau, \mathscr{\beta}\right), t-\tau \geq \Gamma_{0} .
$$

Thus, for any $M \geq M\left(\eta_{0}, \tau, \mathscr{\beta}\right)$ and $t-\tau \geq \Gamma_{0}$, we have

$$
\frac{\mathrm{d}}{\mathrm{d} t} \sum_{|m| \geq 2 M}\left\|\phi_{m}(t)\right\|_{H_{\gamma}}^{2} \leq-\delta_{0} \sum_{|m| \geq 2 M}\left\|\phi_{m}(t)\right\|_{H_{\gamma}}^{2}+\frac{2 \chi_{0}}{M}\|\phi(t)\|_{H_{\gamma}}^{2} .
$$

By (3.13), it can easily obtain

$$
\|\phi(t)\|_{H_{\gamma}}^{2} \leq \mathrm{e}^{2\left(C_{0} R_{0}^{2}-\delta_{0}\right)(t-\tau)}\|\phi(\tau)\|_{H_{\gamma}}^{2}
$$

From (3.19) and (3.20), we get

$$
\frac{\mathrm{d}}{\mathrm{d} t} \sum_{|m| \geq 2 M}\left\|\phi_{m}(t)\right\|_{H_{\gamma}}^{2} \leq-\delta_{0} \sum_{|m| \geq 2 M}\left\|\phi_{m}(t)\right\|_{H_{\gamma}}^{2}+\frac{2 \chi_{0}}{M} \mathrm{e}^{2\left(C_{0} R_{0}^{2}-\delta_{0}\right)(t-\tau)}\|\phi(\tau)\|_{H_{\gamma}}^{2} .
$$

Furthermore, by Gronwall's inequality, we have

$$
\sum_{|m| \geq 2 M}\left\|\phi_{m}(t)\right\|_{H_{\gamma}}^{2} \leq\left(\mathrm{e}^{-\delta_{0}(t-\tau)}+\frac{2 \chi_{0}}{M} \cdot \frac{\mathrm{e}^{\left(2 C_{0} R_{0}^{2}-\delta_{0}\right)(t-\tau)}-1}{2 C_{0} R_{0}^{2}-\delta_{0}}\right)\|\phi(\tau)\|_{H_{\gamma}}^{2} .
$$

Set

$$
\begin{aligned}
& \tilde{M}=\max \left\{M\left(\eta_{0}, \tau, \mathscr{\beta}\right), \frac{2 \chi_{0}\left(\mathrm{e}^{\left(2 C_{0} R_{0}^{2}-\delta_{0}\right) \Gamma_{0}}-1\right)}{\left(1-\mathrm{e}^{-\delta_{0} \Gamma_{0}}\right)\left(2 C_{0} R_{0}^{2}-\delta_{0}\right)}+1\right\}, \\
& \Gamma_{0}=\Gamma_{0}\left(\eta_{0}, \tau, \mathscr{\beta}\right)>T(\tau, \mathscr{\beta}),
\end{aligned}
$$


and define $H_{2 \tilde{M}}=\left\{\phi=\left(\phi_{m}\right)_{m \in \mathbf{Z}} \in H_{\gamma}\left|\phi_{m}=0,\right| m \mid>2 \tilde{M}\right\}$, it is clear that $\operatorname{dim} H_{2 \tilde{M}}=(4 \tilde{M}+1)^{4}<+\infty$. Let $\mathscr{P}: H_{\gamma} \rightarrow H_{2 \tilde{M}}$ be the finite dimensional orthoprojector from $H_{\gamma}$ to $H_{2 \tilde{M}}$, then for $t=\Gamma_{0}+\tau>\tau$, (3.14) holds with

$$
0<\tilde{\eta}=\left(\mathrm{e}^{-\delta_{0} \Gamma_{0}}+\frac{2 \chi_{0}}{\tilde{M}} \cdot \frac{\mathrm{e}^{\left(2 C_{0} R_{0}^{2}-\delta_{0}\right) \Gamma_{0}}-1}{2 C_{0} R_{0}^{2}-\delta_{0}}\right)^{1 / 2}<1,
$$

where $R_{0}, \delta_{0}, C_{0}$ and $\tilde{M}, \Gamma_{0}$ as in (3.3), (3.4), (3.7) and (3.21), respectively. The proof is completed.

As a straightforward consequence of Lemma 2.1, Lemma 3.1 and Lemma 3.2, we get the following Theorem 3.1.

Theorem 3.1. The compact kernel sections $\mathcal{K}(\tau)$ has a finite fractal dimension $\operatorname{dim}_{f} \mathcal{K}(\tau)$, which satisfies

$$
\operatorname{dim}_{f} \mathcal{K}(\tau) \leq(4 \tilde{M}+1)^{4} \cdot \ln \left(1+\frac{8\left(1+\mathrm{e}^{\left(C_{0} R_{0}^{2}-\delta_{0}\right) \Gamma_{0}}\right)}{1-\tilde{\eta}}\right) \cdot\left(\ln \frac{2}{1+\tilde{\eta}}\right)^{-1}
$$

where $R_{0}, \delta_{0}, C_{0}, \tilde{M}, \Gamma_{0}$ and $\tilde{\eta}$ as in (3.3), (3.4), (3.7), (3.21) and (3.22), respectively.

\section{Conclusions}

This paper studied the fractal dimension of the compact kernel sections which is generated by the process of the dissipative non-autonomous KGS lattice system described in (3.1) by applying a criterion given in Lemma 2.1 cited directly from Zhou et al. [21], and then an upper bound of the fractal dimension is obtained in (3.23) presented in Theorem 3.1.

Remark. We can use the argument in this paper to study the dissipative non-autonomous Klein-Gordon-Schrödinger lattice system defined on $\mathbf{Z}^{n}$ with $n \geq 2, n \in \mathbf{N}$. In this case, operator A possesses the following decomposition

$$
A=A_{1}+A_{2}+\cdots+A_{n},
$$

meanwhile

$$
A_{j}=B_{j} \bar{B}_{j}=\bar{B}_{j} B_{j}, \quad\left\|B_{j}\right\|_{H} \leq K, \quad j=1,2, \cdots, n,
$$

where $\|\cdot\|_{H}$ means the norm in space $H, K$ is a positive constant. Here, linear operator $B_{j}: H \rightarrow H\left(H=\ell^{2}\right.$ or $\left.l^{2}\right)$ and its adjoint operator $\bar{B}_{j}$ are defined by

$$
\left(B_{j} z\right)_{m}=\sum_{k=-m_{0}}^{m_{0}} b_{j, k} z_{m_{j k}}, \quad\left(\bar{B}_{j} z\right)_{m}=\sum_{k=-m_{0}}^{m_{0}} b_{j,-k} z_{m_{j k}}, \quad z=\left(z_{m}\right)_{m \in \mathbf{Z}^{n}} \in H,
$$

where $j=1,2, \cdots, n$ and $m_{j k}=\left(m_{1}, m_{2}, \cdots, m_{j-1}, m_{j}+k, m_{j+1}, \cdots, m_{n}\right) \in \mathbf{Z}^{n}$.

\section{Acknowledgements}

The author would like to thank the anonymous referees for their helpful comments and thank the editors for their help. 


\section{Conflicts of Interest}

The author declares no conflicts of interest regarding the publication of this paper.

\section{References}

[1] Cheban, D.N., Kloeden, P.E. and Schmalfuß, B. (2002) The Relationship between Pullback, Forward and Global Attractors of Non-Autonomous Dynamical Systems. Nonlinear Dynamical Systems Theory, 2, 9-28.

[2] Li, Y., Zha, T., Wu, H. and Wei, J. (2016) Pullback Exponential Attractors for Non-Autonomous Dynamical System in Space of Higher Regularity. Journal of Applied Analysis and Computation, 6, 242-253.

[3] Wang, Y., Li, D.S. and Kloeden, P.E. (2004) On the Asymptotic Behaviour of Non-Autonomous Dynamical Systems. Nonlinear Analysis TMA, 59, 35-53. https://doi.org/10.1016/j.na.2004.03.035

[4] Zhou, S. and Wang, L.S. (2003) Kernel Sections for Non-Autonomous Damped Wave Equations with Critical Exponent. Discrete and Continous Dynamical Systems, 9, 399-412. https://doi.org/10.1090/qam/2019621

[5] Zhou, S. (2003) Kernel Sections for Damped Non-Autonomous Wave Equations with Memory and Critical Exponent. Quarterly of Applied Mathematics, 61, 731-757. https://doi.org/10.1090/qam/2019621

[6] Bates, P.W., Lisei, H. and Lu, K. (2006) Attractors for Stochastic Lattice Dynamical Systems. Stochastics and Dynamics, 6, 1-21. https://doi.org/10.1142/S0219493706001621

[7] Gu, A., Zhou, S. and Jin, Q. (2015) Random Attractors for Partly Dissipative Stochastic Lattice Dynamical Systems with Multiplicaive White Noises. Acta Mathematicae Applicatae Sinica (English Series), 31, 567-576.

https://doi.org/10.1007/s10255-015-0486-0

[8] Jia, Q., Zhou, S. and Yin, F. (2003) Kolmogorov Entropy of Global Attrator for Dissipative Lattice Dynamical Systems. Journal of Mathematical Physics, 44, 5804-5810. https://doi.org/10.1063/1.1626269

[9] Li, X.J. and Zhong, C.K. (2005) Attractors for Partly Diassipative Lattice Dynamical Systems in $\ell^{2} \times \ell^{2}$. Journal of Computational and Applied Mathematics, 177, 159-174. https://doi.org/10.1016/j.cam.2004.09.014

[10] Li, X.J. and Wang, D.B. (2007) Attractors for Partly Diassipative Lattice Dynamical Systems in Weighted Spaces. Journal of Mathematical Analysis and Application, 325, 141-156. https://doi.org/10.1016/j.jmaa.2006.01.054

[11] Wang, B. (2006) Dynamics of Systems on Infinite Lattices. Journal of Differential Equations, 221, 224-245. https://doi.org/10.1016/j.jde.2005.01.003

[12] Zhou, S. (2002) Attractors for Lattice Systems Corresponding to Evolution Equation. Nonlinearity, 15, 1079-1095. https://doi.org/10.1088/0951-7715/15/4/307

[13] Zhou, S. (2002) Attractors for Second-Order Lattice Dynamical Systems. Journal of Differential Equations, 179, 605-624. https://doi.org/10.1006/jdeq.2001.4032

[14] Zhou, S. (2003) Attractors for First-Order Dissipative Lattice Dynamical Systems. Physica D, 178, 51-61. https://doi.org/10.1016/S0167-2789(02)00807-2

[15] Zhou, S. (2004) Attractors and Approximation for Lattice Dynamical Systems. Journal of Differential Equations, 200, 342-368. https://doi.org/10.1016/j.jde.2004.02.005

[16] Zhou, S. and Shi, W. (2006) Attractors and Dimension of Dissipative Lattice Sys- 
tems. Journal of Differential Equations, 224, 172-204.

https://doi.org/10.1016/j.jde.2005.06.024

[17] Zhou, S. (2008) Fractal Dimension of Global Attractors for Some Dissipative Lattice Systems. International Journal of Bifurcation and Chaos, 18, 3447-3454. https://doi.org/10.1142/S0218127408022482

[18] Wang, B. (2007) Asymptotic Behaviour of Non-Autonomous Lattice Systems. Journal of Mathematical Analysis and Application, 331, 121-136. https://doi.org/10.1016/j.jmaa.2006.08.070

[19] Zhao, X. and Zhou, S. (2008) Kernel Sections for Process and Non-Autonomous Lattice Systems. Discrete and Continous Dynamical Systems (Series B), 9, 763-785. https://doi.org/10.3934/dcdsb.2008.9.763

[20] Zhou, S., Zhao, C. and Liao, X. (2007) Compact Uniform Attractors for Dissipative Non-Autonomous Lattice Dynamical Systems. Communications on Pure and Applied Analysis, 6, 1087-1111. https://doi.org/10.3934/cpaa.2007.6.1087

[21] Zhou, S., Zhou, C. and Wang, Y. (2008) Finite Dimensionality and Upper Semocontinuity of Compact Kernel Section of Non-Autonomous Lattice Systems. Discrete and Continous Dynamical Systems, 21, 1259-1277. https://doi.org/10.3934/dcds.2008.21.1259

[22] Zhou, S., Huang, J. and Han, X. (2010) Compact Kernel Sections for Dissipative Non-Autonomous Zakharov Equation on Infinite Lattices. Communications on Pure and Applied Analysis, 9, 193-210. https://doi.org/10.3934/cpaa.2010.9.193

[23] Zhou, S. and Han, X. (2012) Pullback Exponential Attractors for Non-Autonomous Lattice Systems. Journal of Dynamics and Differential Equations, 24, 601-631. https://doi.org/10.1007/s10884-012-9260-7

[24] Abdallah, A.Y. (2006) Asymptotic Bahaviour of the Klein-Gordon-Schrödinger Lattice Dynamical Systems. Communications on Pure and Applied Analysis, 5, 55-69. https://doi.org/10.3934/cpaa.2006.5.55

[25] Abounouh, M., Goubet, O. and Hakim, A. (2003) Regularity of the Attractor for a Coupled Klein-Gordon-Schrödinger Systems. Differential Integral Equations, 16, 573-581.

[26] Yin, F., Zhou, S., Yin, C. and Xiao, C. (2007) Global Attractor for Klein-GordonSchrödinger Lattice Systems. Applied Mathematics and Mechanics (English Edition), 28, 695-706. https://doi.org/10.1007/s10483-007-0514-y

[27] Abdallah, A.Y. (2015) Uniform Exponential Attractors for Non-Autonomous Klein-Gordon-Schrödinger Lattice Systems in Weighted Spaces. Nonlinear Analysis. Theory, Methods \& Applications, 127, 279-297. https://doi.org/10.1016/j.na.2015.07.013

[28] Huang, J., Han, X. and Zhou, S. (2009) Uniform Attractors for Non-Autonomous Klein-Gordon-Schrödinger Lattice Systems. Applied Mathematics and Mechanics (English Edition), 30, 1597-1607. https://doi.org/10.1007/s10483-009-1211-Z

[29] Zhao, C. and Zhou, S. (2007) Compact Kernel Sections for Non-Autonomous Klein-Gordon-Schrödinger Equation on Infinite Lattices. Journal of Mathematical Analysis and Application, 332, 32-56. https://doi.org/10.1016/j.jmaa.2006.10.002

[30] Zhou, S. and Han, X. (2013) Uniform Exponential Attractors for Non-Autonomous KGS and Zakharov Lattice Systems with Quasiperiodic External Forces. Nonlinear Analysis: Theory, Methods \& Applications, 78, 141-155.

https://doi.org/10.1016/j.na.2012.10.001 